\title{
Neurogene Störungen der Sprache und des Sprechens
}

Annette Baumgärtner, Anja Staiger

\author{
Neurologische Erkrankungen können zu Störungen der Kommunikationsfähigkeit \\ führen. In diesem Beitrag werden Grundlagen zu den neurogenen Störungen der \\ Sprache (Aphasien) und des Sprechens (Sprechapraxie, Dysarthrie) vermittelt und \\ Möglichkeiten zur Diagnostik und Behandlung aufgezeigt.
}

\section{Einleitung}

Neurologische Erkrankungen führen in vielen Fällen zu Störungen der Sprache und des Sprechens. Störungen der Sprache (Aphasien) betreffen - in unterschiedlichsten Ausprägungen - die Vorgänge des Verstehens und Formulierens sprachlicher Äußerungen (Wortbedeutung, Grammatik, Lautstruktur). Auch sind in der Regel die schriftsprachlichen Leistungen Lesen und Schreiben beeinträchtigt.

Neurogene Störungen des Sprechens umfassen die Mechanismen der motorischen Planung (Sprechapraxie) oder der Ausführung von Sprechbewegungen, z. B. infolge von Parese oder Ataxie der am Sprechen beteiligten Muskulatur (Dysarthrien). Diese Störungen betreffen ausschließlich die Sprachproduktion. Das Verstehen von Sprache sowie die schriftsprachlichen Leistungen (Lesen, Schreiben) sind nicht betroffen.

Merke

Aphasie, Sprechapraxie und Dysarthrie können assoziiert auftreten.

Störungen der Sprache und des Sprechens führen zu teils gravierenden Einschränkungen in der Kommunikationsfähigkeit. Unterhaltungen können beispielsweise dadurch beeinträchtigt sein, dass die Patienten Gesprächsinhalte nicht mehr verstehen können oder ihre Mitteilungsabsichten aufgrund von Wortfindungsstörungen oder motorischen Einschränkungen beim Sprechen nicht mehr ausreichend übermitteln können.
Da sprachlich-kommunikative Kompetenzen von zentraler Bedeutung für nahezu alle Lebensbereiche sind, haben Aphasien und neurogene Sprechstörungen weitreichende Auswirkungen auf die gesellschaftliche Teilhabe und die selbstständige Lebensführung. Einschränkungen in der Kommunikationsfähigkeit werden von Patienten in der Regel als äußerst belastend und ihre Auswirkungen gegenüber Störungen der Extremitätenmotorik oftmals sogar als schwerwiegender empfunden. Betroffene beschreiben angesichts der Unfähigkeit, sich zu verständigen, intensive Frustration, Hoffnungslosigkeit, Isolation und Depression [1].

$\begin{array}{ll}\text { ABKÜRZUNGEN } \\ \text { AAC } & \text { augmentative and alternative communication } \\ \text { AAT } & \text { Aachener Aphasietest } \\ \text { DGN } & \text { Deutsche Gesellschaft für Neurologie } \\ \text { EMG } & \text { Elektromyografie } \\ \text { EPG } & \text { Elektropalatografie } \\ \text { ESKOPA-TM } & \text { Evidenzbasierte sprachsystematische und } \\ & \text { kommunikativ-pragmatische Aphasietherapie } \\ \text { FTLD } & \text { frontotemporale Lobärdegeneration } \\ \text { ILAT } & \text { Intensive Language Action Therapy } \\ \text { LSVT } & \text { Lee-Silverman Voice Treatment } \\ \text { MS } & \text { Multiple Sklerose } \\ \text { PPA } & \text { primär progressive Aphasie } \\ \text { PROMPT } & \text { Prompts for Restructuring Oral Muscular Phone- } \\ & \text { tic Targets } \\ \text { SMA } & \text { supplementärmotorisches Areal } \\ \text { tDCS } & \text { transkranielle Gleichstromstimulation } \\ \text { THS } & \text { tiefe Hirnstimulation } \\ \text { TMS } & \text { transkranielle Magnetstimulation } \\ \text { VO } & \text { Verordnung }\end{array}$




\section{Neurogene Störungen der Sprache (Aphasien) \\ Begriffsbestimmung}

\section{DEFINITION}

Aphasie

Aphasien sind erworbene Sprachstörungen, die nach abgeschlossenem Spracherwerb infolge einer Schädigung sprachrelevanter Areale im Gehirn auftreten. Die Störungen betreffen alle expressiven und rezeptiven sprachlichen Fähigkeiten, also Sprechen und Schreiben ebenso wie auditives Verstehen und Lesen, wenn auch individuell in unterschiedlichem Ausmaß. Die Betroffenen haben weder eine Denkstörung noch eine Intelligenzminderung, sondern sie haben Probleme, ihre Gedanken in eine sprachliche Form zu bringen und sprachliche Informationen zu entschlüsseln.

\section{Merke}

Trotz der für Aphasie typischen Schwierigkeiten, gesprochene und geschriebene Sprache zu verstehen, und verbal mündlich oder schriftlich zu kommunizieren, erfassen die Betroffenen i. d. R. den situativen Kontext adäquat und zeigen z. B. durch begleitende Mimik oder Gestik eine eindeutige Mitteilungsabsicht.

\section{Häufigkeit}

Angaben zur Prävalenz von Aphasien aller Ätiologien schwanken stark. Während man in Deutschland von ca. 100 pro 100000 ausgeht [2], werden in den USA ca. 400 pro 100.000 angegeben [3]. Die Inzidenz zerebrovaskulär bedingter behandlungsbedürftiger Aphasien in Deutschland wird mit ca. 25000 pro Jahr angegeben [2]. Etwa 30-40\% aller Schlaganfallpatienten haben initial eine Aphasie.

\section{Ätiologie}

Etwa $80 \%$ aller Aphasien sind Folge eines Schlaganfalls, bei dem perisylvische sprachrelevante Areale der sprachdominanten (meist der linken) Hemisphäre geschädigt wurden. Die restlichen 20\% der Aphasien sind durch Hirnblutungen, Schädel-Hirn-Traumata, Hirntumoren, entzündliche Erkrankungen des Gehirns oder durch Epilepsie bedingt.

Einen Sonderfall bilden die Aphasien aufgrund umschriebener degenerativer Prozesse in der sprachdominanten Hemisphäre. Diese sogenannten primär progressiven (auch progredienten) Aphasien (PPA) stellen eine Subgruppe der frontotemporalen Demenzen dar. PPA sind durch einen schleichenden Beginn mit fortschreitender
Verschlechterung einer für die ersten 1-2 Jahre isoliert auftretenden Sprachstörung gekennzeichnet.

\section{Krankheitsverlauf und Prognose}

Der Krankheitsverlauf einer Aphasie ist abhängig von der Grunderkrankung. Im Akutstadium des Schlaganfalls fluktuiert die aphasische Symptomatik aufgrund der sich rasch ändernden pathophysiologischen Prozesse stark. In der frühen Phase nach Schlaganfall bestimmen Lokalisation, Ausmaß und Art des Hirninfarktes (ischämisch oder hämorrhagisch) sowie mögliche Komplikationen durch Störung elementarer Hirnfunktionen den Verlauf der Funktionserholung [4]. 4 Wochen nach dem Ereignis haben sich bei ca. einem Drittel der Patienten mit initialer Aphasie die Sprachfunktionen weitgehend normalisiert. Innerhalb von 6 Monaten nach Insult bildet sich die Symptomatik abhängig von Lokalisation, Volumen und Reversibilität der Läsion bei ca. $50 \%$ der initial aphasischen Patienten vollständig zurück [5]. Danach flacht die Kurve der Spontanrückbildung zunehmend ab.

\section{Merke}

Der initiale Schweregrad der Aphasie ist ein wichtiger Prädiktor für die Rückbildung der aphasischen Symptomatik nach Schlaganfall.

Initial leichtgradige Aphasie bilden sich häufig vollständig zurück. Jedoch ist auch bei anfänglich schweren Aphasien ein günstiger Verlauf möglich [6]. Im chronischen Stadium zeigen aphasische Patienten mit leichterem Schweregrad des Schlaganfalls einen größeren Behandlungserfolg als schwerer betroffene Patienten [7].

Das Lernpotenzial chronisch aphasischer Patienten hängt zudem von der Integrität des Hippokampus der sprachdominanten Hemisphäre und seiner umgebenden Marklagerstrukturen ab [8]. Weitere patientenoder schlaganfallbezogene Faktoren (wie Zahl der vorherigen Schlaganfälle) beeinflussen das Verbesserungspotenzial nicht [7].

\footnotetext{
Merke

Das therapeutische Potenzial bei vaskulär bedingten Aphasien ist unabhängig von Alter, Geschlecht, Bildungsniveau, oder Krankheitsdauer.
}

\section{Klassifikation}

Eine landläufige Unterteilung besonders im Akutstadium ist die in „flüssige“ bzw. „nicht-flüssige“ Aphasien. Ohne weitere Spezifizierung ist der Begriff der „Flüssigkeit“ jedoch mehrdeutig, denn die beobachtete „Unflüssigkeit" kann durch Schwierigkeiten der Wortfindung und/oder des Satzbaus über Probleme bei der Planung von Sprechbewegungen (s.u., Sprechapraxie) bis hin zu zentral oder peripher bedingten Störungen 
des Sprechens (s. u., Dysarthrien) verursacht sein. Differenzialdiagnostisch ist die Bezeichnung „unflüssige Aphasie" daher unzureichend. Auch die Bezeichnung „flüssige Aphasie“ ist bezüglich weiterführender Diagnostik und Therapie wenig aussagekräftig.

Genauer, da sie bereits grobe Hinweise auf SymptomCluster enthält, ist die traditionelle Unterteilung der Aphasien in 4 Standardsyndrome, bei denen Sprachproduktions- und Sprachverständnisprobleme in unterschiedlichem Maß gestört sind (s. - Abb. 1).

Die Klassifikation nach Syndromen ist für die schnelle Informationsweitergabe im klinischen Alltag nützlich. Für die Planung einer Sprachtherapie ist sie jedoch nur eingeschränkt geeignet, da

- nicht alle Aphasien eindeutig einem bestimmten Syndrom zugeordnet werden können;

- sich die Klassifikation einer Aphasie durch Spontanremission ändern kann;

- bestimmte Symptome, wie beispielsweise Fehler der Lautstruktur, nicht nur bei einem, sondern bei mehreren Syndromen zu beobachten sind (wie z. B.

Lautfehler, die sowohl bei Broca- als auch bei Wernicke-Aphasie auftreten).

Für die Therapieplanung wird in jedem Fall ein individuelles Profil der sprachlichen Leistungen und Störungen in den unterschiedlichen linguistischen Ebenen wie Lautstruktur, Wortschatz, Verarbeitung von Wort- und Satzbedeutung und Grammatik benötigt (s.u.).

\section{HINTERGRUNDINFORMATION \\ Die sogenannte motorische Aphasie}

Der Begriff der motorischen Aphasie ist geschichtlich bedingt. Er ist aus heutiger Sicht irreführend, da er fälschlicherweise impliziert, dass - eine Bewegungs-/Sprechstörung vorliegt und

- das rezeptive Sprachverständnis intakt ist.

Zur schnellen Informationsweitergabe ist daher der Begriff „Broca-Aphasie“ vorzuziehen. Für den Begriff „sensorische Aphasie“ gelten ähnliche Bedenken.

Die primär progressiven Aphasien werden in 3 Unterformen eingeteilt:

- die agrammatische (auch nicht-flüssige) Variante,

- die semantische Variante,

- die logopenische Variante.

Die drei Varianten können jeweils relativ spezifischen neuronalen Substraten (nämlich frontalen, anterior temporalen und temporo-parietalen Arealen) zugeordnet werden.

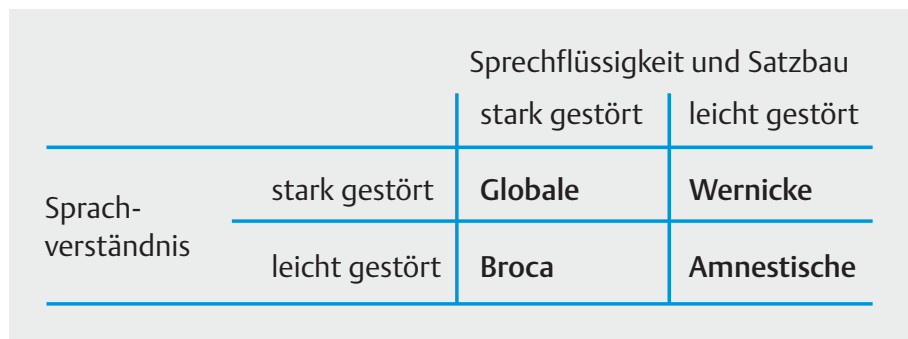

Abb.1 Klassifikationsschema der aphasischen Standardsyndrome (vereinfachte Darstellung in Anlehnung an den Aachener Aphasietest/AAT; [9]).

\section{Erscheinungsbild}

Bei Aphasie sind die verschiedenen Komponenten des Sprachsystems individuell in unterschiedlichem Maß betroffen. Die zentralen Komponenten (oder „linguistischen Ebenen") und Beispiele für auf dieser Ebene auftretenden aphasischen Symptome sind

- Phonologie (Lautstruktur),

- Abweichungen in Auswahl und Reihenfolge der Sprachlaute (z. B. sprötzlich für plötzlich),

- Morphologie (Wortstruktur),

- Fehler bei den kleinsten bedeutungstragenden Elementen eines Wortes (z. B. „dann bin ich zur Untersuch" statt Untersuchung),

- Lexikon (Wortschatz),

- Probleme beim Wortabruf (deutlich z. B. durch sprachliches Suchverhalten wie beim Benennen „Haare machen ... kämmen ... Kamm“),

- Semantik (Bedeutungsstruktur),

- Fehler in der Verwendung von Bedeutungseinheiten (z. B. „Hüter des Lichts“ statt Elektriker),

- Syntax (Grammatik),

- Abweichungen im Satzbau (z. B. Auslassung grammatischer Elemente bei Agrammatismus wie „...Hirnschlag ... und ohnmächtig ... Krankenhaus")

Abhängig vom generellen Schweregrad einer Aphasie kann die aphasische Symptomatik auf den verschiedenen linguistischen Ebenen unterschiedlich stark bis hin zu schwersten Störungen ausgeprägt sein.

Neben den Symptomen auf den linguistischen Ebenen treten häufig sprachlich-repetitive Phänomene wie Perseveration (ungewolltes „Hängenbleiben“ an bereits produzierten sprachlichen Einheiten), Echolalie (unkontrollierte Wiederholung von unmittelbar vorangegangenen Äußerungen oder Äußerungsanteilen des Gesprächspartners) oder recurring utterances (aus immer wiederkehrenden Silben, Wörtern, oder Phrasen bestehende Automatismen) auf. Zudem sind oft eine gegenüber der Sprache von gesunden Sprechern erhöhte Anzahl an Redefloskeln und stereotypen Äußerungen zu beobachten. 
FALLBEISPIEL

\section{Fall 1: Aphasie}

Frau B., tätig im Personalmanagement einer großen Kosmetikfirma, erleidet 43-jährig eine intrazerebrale temporale Blutung links. Es resultiert eine mittel- bis schwergradige Wernicke-Aphasie. In der Spontansprache fallen eine überschießende Sprachproduktion mit vielen Satzteilverdoppelungen und grammatischen und semantischen Fehlern auf.

Gefragt wie alles angefangen hat, antwortet Frau B. deutlich artikuliert und mit schneller Sprechgeschwindigkeit: „Also ich war am Sonntag weiss ich auch gar nicht so recht was mir passiert ich war dann sofort weg von allen Gedanken dann wurde ich am Sonntag wurde ich niederhingelegt wurde ich dann also eingeweiht einbeschrankt niedergelegt worden sind...". Das Sprachverständnis ist stark beeinträchtigt; Frau B. kann Unterhaltungen kaum folgen. Initial kann sie ihre sprachliche Symptomatik nicht wahrnehmen; sie bemerkt jedoch, dass etwas mit ihrer Sprache „nicht stimmt“.

Im Verlauf von 2 Jahren, in denen sie Sprachtherapie erhält, reduziert sich die sprachliche Symptomatik; das Syndrom wird nun als amnestische Aphasie klassifiziert. Selbstwahrnehmung und Selbstregulierung der sprachlichen Symptomatik haben sich verbessert.

Frau B. arbeitet stundenweise wieder in ihrem Unternehmen, kann jedoch aufgrund der immer noch bestehenden Aphasie ihre Hauptaufgabe als Schulungsleiterin nicht mehr erfüllen. Seit einigen Wochen berät sie ehrenamtlich im Krankenhaus Patienten mit Aphasie und ihre Angehörigen.

Die Betroffenen nehmen häufig das Ausmaß ihrer sprachlichen Beeinträchtigungen nur eingeschränkt wahr. Selbstkorrekturversuche sind prognostisch günstig, da sie auf eine erhaltene Störungswahrnehmung hinweisen.

\section{Diagnostisches Vorgehen}

Die spezifische Diagnostik der Aphasie zählt zum Aufgabengebiet der sprachtherapeutischen Berufsgruppen. Sie dient

- im Akutstadium:

- der Feststellung der Stimulierbarkeit sprachlicher Äußerungen,

- der Einschätzung des Sprachverständnisses,

- der Bestimmung der Fähigkeit zu spontansprachlichen Äußerungen,

- der Überprüfung von spontan eingesetzten Kompensationsstrategien und weiteren kommunikativen Ressourcen (Mimik, Gestik, Zeichnen, Schreiben),

- dem Screening neuropsychologischer Begleitstörungen,

- der Identifikation von Sofortmaßnahmen zur Ermöglichung der Vermittlung von Grundbedürfnissen und von basaler Kommunikation,
- im subakuten bzw. chronischen Stadium:

- dem Nachweis einer Aphasie,

- der Bestimmung des Schweregrades der Aphasie,

- der Bestimmung des Störungsprofils durch die Identifikation der sprachlichen Beeinträchtigung in den verschiedenen linguistischen Ebenen (Phonologie, Lexikon/Semantik, Grammatik),

- der Identifikation von repetitiven Phänomenen und automatisierten Sprachanteilen,

- der Einschätzung von Selbstwahrnehmung und kompensatorischen Strategien,

- der Planung sprachtherapeutischer Versorgungsmaßnahmen,

- der Verlaufskontrolle.

\section{Anamnese}

Erkrankungsverlauf: Anamnestische Angaben zur Grunderkrankung und ihrem Verlauf stecken den Rahmen für die diagnostischen Ziele und eine sprachtherapeutische Versorgung ab. Diese Informationen sind auch der Krankenakte zu entnehmen.

- Grunderkrankung, Erkrankungsdauer,

- Beginn der Sprachstörung und Verlaufsdynamik (Progredienz, Remission),

- (soweit zutreffend) Lokalisation und Umfang der Läsion,

- Medikation,

- neuropsychologische Begleitstörungen

- zusätzliche neurogene Störungen des Sprechens (Sprechapraxie, Dysarthrie, s.u.),

- Aufmerksamkeitsstörungen,

- Störungen von Kurzzeit- oder Arbeitsgedächtnis,

- Störungen exekutiver Funktionen u. a.,

- neurologische Begleitstörungen (z. B. Hemiparese, Schluckstörung, visuelle Beeinträchtigungen),

- psychische Begleitstörungen (vor allem Depression).

Beeinflussende Faktoren: Verschiedene mit der neurologischen Erkrankung nicht assoziierte Faktoren können das Störungsbild einer Aphasie beeinflussen:

- Hörverlust,

- Mehrsprachigkeit (Deutsch als Zweitsprache),

- vorbestehende Lernbehinderung oder Sprachentwicklungsstörung,

- Analphabetismus,

- Legasthenie.

Partizipation und psychosoziale Faktoren: Für die Planung einer sprachtherapeutischen Intervention sind folgende Informationen über die individuellen Einschränkungen der Partizipation und die psychosozialen Ressourcen eines Patienten von Bedeutung:

- Einfluss der Sprachstörung auf die selbstständige Lebensführung,

- soziale/familiäre Einbettung,

- berufliche Anforderungen an die Sprachfähigkeit, 
- Selbstwahrnehmung der sprachlichen Schwierigkeiten,

- Einsatz von Kompensationsstrategien.

Gegebenenfalls können strukturierte Leitfäden und Fragebögen zur Selbsteinschätzung der Lebensqualität und der subjektiv besonders beeinträchtigenden sprachlichen Schwierigkeiten verwendet werden.

\section{Strukturierte sprachtherapeutische Befundung}

Die Befundung einer Aphasie beinhaltet i.d.R. eine Analyse der Spontansprache und eine Bestimmung der Fehlertypen bei spezifischen sprachlichen Aufgaben (Nachsprechen, Benennen, Schreiben/Lesen, Sprachverständnis) zur Identifizierung gestörter und erhaltener sprachlicher Prozesse.

Je nach Erkrankungsphase unterscheidet sich zusätzlich das spezifische Vorgehen in der Diagnostik wie folgt:

- im Akutstadium (z. B. $[10,11])$ :

- Überprüfung des Sprachverständnisses durch Aufforderung zu Blick- und Kopfbewegungen,

- Überprüfung von Artikulation und Phonation,

- Überprüfung der sprachlichen Stimulierbarkeit durch (Mit-)Singen, Reihensprechen (Zahlen, Wochentage) und/oder Produzieren von Floskeln (z.B. „Macht nichts“),

- im subakuten/chronischen Stadium (z. B. [9, 12]):

- Aphasie ja/nein,

- Bestimmung des Schweregrades der aphasischen Störung anhand eines Leistungsprofils,

- detaillierte Beschreibung der aphasischen Störung auf verschiedenen sprachlichen Verarbeitungsebenen (Phonologie, Lexikon, Morpho-Syntax, Semantik).

Zur Identifikation modalitätsspezifischer Sprachstörungen, die einzelne sprachliche Komponenten wie z. B. das Lesen betreffen („Dyslexie“), stehen zusätzliche standardisierte Diagnostikinstrumente zur Verfügung. Für die Diagnostik von primär progressiven Aphasien existieren im deutschsprachigen Raum noch keine standardisierten Assessments. Zur Diagnostik werden daher konventionelle Assessmentinstrumente herangezogen. Eine Adaptation eines spezifisch für PPA erstellten Diagnostikinstruments aus dem Englischen ist in Vorbereitung.

\section{Therapie}

Sprachtherapeutische Maßnahmen

\section{Merke}

Das übergeordnete Ziel einer Sprachtherapie bei Aphasie ist es, Betroffene darin zu unterstützen, die Teilhabe an den Lebenssituationen oder Lebensbereichen zu erreichen, die für sie von Bedeutung sind.
Ziel der Therapie ist eine möglichst effektive Kommunikation im Alltag, u.U. mit Einsatz digitaler Hilfsmittel (Tablets, Smartphone-Apps) und somit langfristig eine möglichst weitreichende soziale und berufliche Selbstständigkeit und Reintegration.

Der Goldstandard zur Behandlung einer Aphasie ist die sprachtherapeutische Übungsbehandlung. Dabei wird gemeinsam mit den Betroffenen unter dem Aspekt der kommunikativen Alltagsanforderungen eine Gewichtung der vorliegenden sprachlichen Beeinträchtigungen sowie eventueller behandlungsbedürftiger Begleitstörungen vorgenommen und ein individuell angepasster (ggf. interprofessioneller) Therapieplan festgelegt.

Vorgehensweise: Die Auswahl geeigneter sprachtherapeutischer Vorgehensweisen bei Aphasie ist von individuellen Faktoren bestimmt:

- dem Störungsprofil: Ausprägung und Schweregrad von Störungen auf den verschiedenen linguistischen Ebenen:

- Phonologie,

- Lexikon,

- Morpho-Syntax,

- Semantik,

- neuropsychologischen Begleitstörungen, z. B.

- Sprechapraxie,

- Dysarthrie,

- Aufmerksamkeitsstörungen,

- Gedächtnisstörungen,

- der Grunderkrankung (z. B. non-degenerative Erkrankung versus PPA),

- psychosozialen Faktoren.

Behandlungsziele lassen sich auf verschiedenen Ebenen definieren [13]:

- Funktion: Verbesserung sprachlicher Funktionen (z. B. Verbesserung des auditiven Sprachverständnisses),

- Aktivität und Teilhabe: Verbesserung kommunikativer Fähigkeiten (z. B. Telefongespräche führen können) und damit Verbesserung von Teilhabemöglichkeiten (z. B. wieder in Kundenberatung tätig sein),

- Umweltfaktoren: Substitution und Umweltadaptation, z. B.

- Einsatz unterstützender digitaler Hilfsmittel,

- Beratung und Aufklärung von Betroffenen und Angehörigen,

- Anpassung des sozialen/beruflichen Umfelds an veränderte kommunikative Fähigkeiten.

Allgemeine Therapieprinzipien: Voraussetzung für die Wirksamkeit einer Aphasietherapie sind störungsspezifisches individualisiertes Üben sowie Therapieintervalle mit hoher Therapieintensität [14]. Dabei gelten die Prinzipien: 
- Tab. 1 Inhalte der sprachtherapeutischen Intervention.

\begin{tabular}{|c|c|}
\hline Sprachsystematische Therapie & $\begin{array}{l}\text { Kommunikativ-pragmatische } \\
\text { Therapie }\end{array}$ \\
\hline $\begin{array}{l}\text { Therapieziel: Verbesserung der } \\
\text { gestörten linguistischen Funktion }\end{array}$ & $\begin{array}{l}\text { Therapieziel: Verbesserung der } \\
\text { gestörten Handlungsfähigkeit }\end{array}$ \\
\hline häufig unimodal & multimodal \\
\hline $\begin{array}{l}\text { Trainieren des gestörten sprach- } \\
\text { lichen Mechanismus }\end{array}$ & $\begin{array}{l}\text { Verstärken von Umweg- und } \\
\text { Kompensationsstrategien }\end{array}$ \\
\hline Fokus auf Defizit & Fokus auf Ressourcen \\
\hline Linguistische Strukturen & Alltagsrelevante Situationen \\
\hline
\end{tabular}

- Sprachverständnis vor Sprachproduktion,

- Hemmen bei überschießender Sprachproduktion oder Sprachautomatismen,

- Verbesserung der Selbstwahrnehmung für sprachliche Schwierigkeiten,

- multimodale (visuelle, auditive) sprachliche Stimulierung,

- gradueller Einsatz spezifischer sprachlicher Hilfen,

- Übungsaufgaben zu Auswahl, Abgleich, und Differenzierung sprachlicher Items,

- repetitives Üben.

Generell enthält eine sprachtherapeutische Intervention sowohl sprachsystematische als auch kommunikativ-pragmatische Übungsinhalte ( $\triangleright$ Tab. 1$)$.

Phasenspezifische Therapie: Je nach Krankheitsphase ergeben sich spezifische Therapieziele, nämlich

- im Akutstadium:

- Hemmung ggf. vorliegender sprachlicher Automatismen,

- abhängig von Allgemeinzustand und ausreichender Aufmerksamkeit: sprachliche Stimulierung über rhythmisch-melodische Muster und Vor-, Mit-, Nachsprechen von Interjektionen, Redefloskeln, automatisierte Wortreihen; Satzergänzungsaufgaben, etc.

- Verbesserung des Sprachverständnisses;

- im subakuten bzw. chronischen Stadium: individualisierte Therapie unter Berücksichtigung von
- sprachlichem Störungsprofil,

- kognitiven Begleitstörungen,

- kommunikativen Wünschen/Erfordernissen,

- individuellen Lern- und Kompensationsmöglichkeiten für die sprachliche Kommunikation.

Therapie bei primär progressiver Aphasie: Sprachtherapeutische Ansätze bei PPA sind zurzeit Gegenstand intensiver Forschung. Im Vordergrund der Therapie steht, die sprachlich-kommunikativen Fähigkeiten aufrecht zu erhalten, und die Betroffenen darin zu unterstützen, so lange wie möglich ihre Unabhängigkeit zu bewahren.

\section{PRAXIS}

Tipp

Fast alle von PPA betroffenen Patienten können von einer „präventiven“ Sprachtherapie profitieren. Daher sollten die Patienten nach Diagnosestellung möglichst schnell Sprachtherapie erhalten, damit die Zielfindung und ein Plan zum therapeutischen Vorgehen möglichst noch gemeinsam mit den Betroffenen entwickelt werden können [15].

\section{Wirksamkeitsnachweise der Sprachtherapie}

Wirksamkeitsnachweise liegen ausschließlich für intensiv (hochfrequent) durchgeführte Therapieformen vor (Übersicht bei [16]). Eine bekannte und in ihrer Wirksamkeit belegte Therapieform ist die Intensive Language Action Therapy/ILAT. Sie wurde aus der Constraintinduced Movement Therapy abgeleitet und von vornherein als Intensivtherapieansatz konzipiert. Es liegen jedoch weitere evidenzbasierte Intensivtherapieansätze wie z. B. computergestütztes Skripttraining vor [16].

Zur Frage der Wirksamkeit einer Aphasietherapie in der frühen Phase nach Schlaganfall liegen, z. T. wegen methodischer Schwächen der Studien (z. B. keine Kontrollgruppe), noch keine eindeutigen Nachweise vor (Übersicht bei [14]). Die Ergebnisse einer methodisch gut kontrollierten 3-armigen Studie (Very Early Rehabilita-

\section{THERAPIE}

\section{Verordnung von Sprachtherapie}

Nach Heilmittelkatalog: Ziffer 2.4 Störungen der Sprache nach Abschluss der Sprachentwicklung, Diagnosegruppe SP5 Aphasien.

- Heilmittel: Sprachtherapie 30, 45 oder 60 Minuten Einzel- oder Gruppentherapie.

- Verordnungsmenge: bis zu 20×/VO. Gesamtverordnungsmenge des Regelfalls: bis zu 60 Einheiten.

- Frequenzempfehlung: 1-3×wöchentlich.
Falls die Versorgungssituation vor Ort es zulässt, sollte die Therapie eher mit höherer als niedriger Frequenz verordnet werden [21]. Mit Vorabzustimmung durch die Kostenträger ist eine Therapie mit einer höheren als der angegebenen Therapieintensität möglich; insbesondere dann, wenn wie üblich nach der Intensivtherapie eine mehrmonatige Therapiepause geplant ist. 
tion in Speech after Stroke/VERSE) werden in naher Zeit erwartet.

Dagegen liegen inzwischen Nachweise zur Wirksamkeit intensiver Sprachtherapie bei chronischer Aphasie vor [7]. Eine mindestens 3-wöchige individualisierte Therapie, die mit einer Frequenz von mindestens 10 Stunden Sprachtherapie pro Woche durchgeführt wurde, zeigte in einer Stichprobe von > 150 Patienten eine signifikante Verbesserung der funktionellen Alltagskommunikation, unabhängig von Krankheitsdauer, Alter oder Geschlecht der Teilnehmer. Diese Therapieform ist für die meisten Patienten mit Aphasie (ausgenommen schwerstgradige und Restaphasie) geeignet.

\section{Merke}

Das in der Studie von den SprachtherapeutInnen verwendete Manual (ESKOPA-TM) 2020 ist im Hogrefe Verlag erschienen.

\section{Pharmakotherapie}

Die Evidenzlage zu einer begleitenden medikamentösen Intervention bei Aphasie ist uneindeutig [17]. Einzelne Studien zum Einsatz von Donezepil und Memantine zeigen einen positiven Effekt auf sprachliche Teilleistungen wie Benennen oder Nachsprechen; weitere Studien, z. B. zu Piracetam, zeigen keine einheitlichen Ergebnisse. Die Auswirkung einer unterstützenden Pharmakotherapie auf die Alltagskommunikation wurde bislang nicht untersucht. Es gibt keine Hinweise darauf, dass eine isolierte Pharmakotherapie bei Aphasie wirksam ist.

\section{Adjuvante Therapie mit Hirnstimulation}

Neuromodulation durch Hirnstimulation ist ein weiterer Ansatz zur Unterstützung der therapieinduzierten plastischen Prozesse durch sprachtherapeutische Intervention bei Aphasie (PPA eingeschlossen; [18,19]). Während bislang nur wenige Studien veröffentlicht wurden, die die transkranielle Magnetstimulation (TMS) begleitend zur Sprachtherapie eingesetzt haben (z.B. [20], mit negativem Ergebnis), hat sich die Zahl der Studien mit adjuvanter transkranieller Gleichstromstimulation (tDCS) in den letzten Jahren deutlich erhöht. Ein aktueller Cochrane-Review [19]) findet keine Evidenz dafür, dass der Einsatz von tDCS die funktionelle Kommunikationsfähigkeit bei Aphasie über die Erfolge der Sprachtherapie hinaus verbessert. Da einzelne Studien jedoch positive Effekte für die Benennleistung bei Aphasie zeigen, fordern die Autoren weitere Untersuchungen der Auswirkung von tDCS auf die funktionelle Kommunikation mit größeren Stichproben. Eine solche Studie (DC Train Aphasia) wurde 2019 an der Universität Greifswald gestartet.

\section{Neurogene Störungen des Sprechens (Dysarthrien, Sprechapraxie)}

Neurogene Sprechstörungen sind Störungen sprechmotorischer Prozesse, die nach einer Schädigung des zentralen oder peripheren Nervensystems auftreten können. Betroffen sind die Prozesse der Planung oder der Ausführung von Sprechbewegungen. Als motorische Störungen werden sie abgegrenzt zu Störungen expressiver und rezeptiver sprachlicher Fähigkeiten (Aphasien). Ferner werden sie unterschieden von Störungen nichtsprachlicher Bewegungen der für das Sprechen relevanten Muskulatur (z. B. Kauen, Schlucken, „Zunge herausstrecken“).

\section{Begriffsbestimmung}

\section{DEFINITION \\ Sprechapraxie}

Die Sprechapraxie ist eine durch erworbene Schädigung des zentralen Nervensystems bedingte Störung der Planung (oder "Programmierung") von Sprechbewegungen. Die Symptomatik der Sprechapraxie lässt sich nicht durch elementarmotorische Störungen wie z.B. Parese, Akinesie oder Ataxie erklären. Im Vordergrund stehen artikulatorische Fehler und ein unflüssiges, angestrengtes Sprechen.

\section{DEFINITION}

Dysarthrie (auch „Dysarthrophonie“)

Als Dysarthrien werden Sprechstörungen bezeichnet, die aus Schädigungen des zentralen oder peripheren Nervensystems resultieren können und durch elementarmotorische Beeinträchtigungen der Ausführung von Sprechbewegungen verursacht werden. Dysarthrien können alle am Sprechen beteiligten Muskelgruppen betreffen (Atem-, Kehlkopf- und supralaryngeale Muskulatur).

\footnotetext{
Sprechapraxie und Dysarthrie führen zu Beeinträchtigungen der Kommunikation, z. B. aufgrund von Einschränkungen der Verständlichkeit, Verlangsamung oder unnatürlicher Sprechweise.
} 


\section{Häufigkeit}

Zur Prävalenz der Sprechapraxie liegen keine gesicherten Daten vor. Unter den Patienten mit vaskulär bedingten Aphasien haben nach einer vorsichtigen Schätzung mehr als ein Drittel auch eine Sprechapraxie [22].

Dysarthrien stellen die häufigste Form neurologisch bedingter Kommunikationsstörungen dar mit einer Prävalenz von > 400 pro 100000 .

\section{Ätiologie und Pathogenese}

Eine Sprechapraxie resultiert zumeist aus Infarkten oder Blutungen im Stromgebiet der vorderen Äste der A. cerebri media links, mit Läsionen im posterioren Anteil des Gyrus frontalis inferior („Broca-Areal“, pars opercularis), des ventrolateralen prämotorischen Kortex einschließlich des angrenzenden motorischen Gesichtskortex und/oder der anterioren Inselrinde der linken Hemisphäre.

Zunehmend wird auch über „progrediente Sprechapraxien “ im Rahmen frontotemporaler Lobärdegenerationen (FTLD), insbesondere der nicht-flüssigen Variante der primär progressiven Aphasien (PPA), berichtet.

Dysarthrien resultieren aus Schädigungen motorischer Areale, die an der Kontrolle von Sprechbewegungen beteiligt sind, also

- des primär-motorischen Kortex (Gesichts- und Larynxregion),

- der absteigenden kortikonukleären Bahnen und der motorischen Kerne im Hirnstamm und im Rückenmark,

- des Kleinhirns (vor allem der wurmnahen Abschnitte der oberen Kleinhirnrinde und des Nucleus dentatus) sowie

- der Basalganglien.

Diese Schädigungen können unter anderem bedingt sein durch

- fokale Gewebeschädigung (z. B. Schädel-HirnTrauma, Schlaganfall, periphere Nervenläsionen),

- neurodegenerative Prozesse (Morbus Parkinson, Morbus Huntington, Motoneuronerkrankungen, hereditäre Ataxien etc.),

- entzündliche oder demyelinisierende Prozesse (z. B. Multiple Sklerose),

- hypoxische Schädigungen,

- neuromuskuläre Erkrankungen (z. B. Myasthenia gravis).

Cave

Bilaterale Schädigungen führen zu schweren und persistierenden Dysarthrien.
Eine Sonderrolle spielen Dysfunktionen mesiofrontaler Rindenareale, insbesondere des supplementärmotorischen Areals (SMA), die zu reduziertem Sprechantrieb und zu Störungen der Sprechinitiierung führen können.

\section{Klassifikation}

Neurogene Sprechstörungen werden traditionell wie folgt klassifiziert:

- Sprechapraxie,

- Dysarthrie:

- peripher-paretische („schlaffe“) Dysarthrie: z. B. bei amyotropher Lateralsklerose, bei peripheren Nervenläsionen oder im Rahmen von Hirnnervensyndromen,

- zentral-paretische („spastische“) Dysarthrie: z. B. bei Zerebralparese, Schlaganfall, MS,

- ataktische Dysarthrie: bei hereditären, erworbenen oder sporadisch-degenerativen Ataxien,

- hypokinetische Dysarthrie: v. a. bei Morbus Parkinson (Prävalenz bis zu 90\%),

- hyperkinetische Dysarthrie: z. B. choreatischhyperkinetisch (v. a. bei Morbus Huntington) oder athetotisch (z. B. bei Zerebralparese).

Darüber hinaus werden den neurogenen Sprechstörungen häufig zugerechnet:

- fokale Dystonien:

- spasmodische Dysphonie (laryngeale Dystonie vom Adduktor-, Abduktor- oder gemischten Typ),

- oromandibuläre Dystonie,

- Stimmtremor, z. B.

- essenzieller Tremor,

- Stimmtremor bei Morbus Parkinson oder bei zerebellärer Ataxie

- erworbenes neurogenes Stottern,

- Störungen des Sprechantriebs (inkl. akinetischer Mutismus), v. a. bei mesiofrontalen Läsionen.

\section{Merke}

Die Klassifikation neurogener Sprechstörungen orientiert sich überwiegend an der Taxonomie der Störungen der Gliedmaßenmotorik und damit an den pathophysiologischen Mechanismen, die der jeweiligen Sprechbewegungsstörung vermutlich zugrunde liegen.

\section{Erscheinungsbild}

Sprechapraxie

Sprechapraktische Störungen ohne zusätzliche aphasische Symptomatik (z.B. Agrammatismus, Wortfindungsstörungen) kommen selten vor. Die sprechapraktische Symptomatik kann jedoch auch im Vordergrund der Kommunikationsstörung stehen. 
Die Sprechapraxie lässt sich auf drei Störungsebenen beschreiben:

- Störungen der Lautbildung,

- Auffälligkeiten im Sprechverhalten und

- Störungen der rhythmisch-melodischen Eigenschaften des Sprechens (Prosodie).

Störungen der Lautbildung: Konsonanten oder Vokale werden

- graduell fehlgebildet („phonetisch entstellt“),

- durch andere Sprachlaute ersetzt (z. B. Frosch $\rightarrow$ Prosch),

- ausgelassen (Frosch $\rightarrow$ _rosch),

- hinzugefügt (Frosch $\rightarrow$ Froksch).

Cave

Das Fehlermuster ist typischerweise durch spezifische sprachliche Eigenschaften beeinflusst (z. B. erhöhte Fehleranfälligkeit bei Silben mit hoher Konsonantendichte wie „Specht“).

Auffälligkeiten im Sprechverhalten: Vor allem

- artikulatorische Such- und Korrekturprozesse (z. B. Frosch $\rightarrow$ Pr.sch. fros ... Pfrosch ... Frosch),

- sicht- und hörbare Sprechanstrengung (z. B. erhöhter Tonus und/oder Mitbewegungen der orofazialen Muskulatur, erhöhte Sprechstimmlage als Folge der Sprechanstrengung),

- Überartikulation (z. B. übergroße Bewegungsamplituden).

Dass die Patienten ihre Lautfehler häufig bereits während der Artikulation bemerken und zu korrigieren versuchen, lässt auf weitgehend intakte sensorische Funktionen schließen.

\section{Störungen der Prosodie: Vor allem}

- verlangsamte Artikulationsbewegungen, verlängerte Lautübergänge,

- Unterbrechungen des Redeflusses durch Fehlversuche und Selbstkorrekturen (s. o., Sprechverhalten),

- monotones, silbisches („Silbe für Silbe“) Sprechen.

\section{Merke}

Das sprechapraktische Störungsmuster ist typischerweise durch eine hohe Variabilität gekennzeichnet (z. B. inkonstante und inkonsistente Lautbildungsfehler). Es können „Inseln störungsfreier Produktion“ auftreten (z. B. bei hochautomatisierten Äußerungen).

\section{Dysarthrie}

Typischerweise sind bei Dysarthrie mehrere oder alle am Sprechen beteiligten Funktionen - Atmung, Stimme und Artikulation - betroffen. Dysarthrien können jedoch auch aus relativ isolierten Funktionsstörungen (z. B. Störungen der lingualen Artikulation) resultieren.

\section{FALLBEISPIEL}

\section{Fall 2: Sprechapraxie}

Herr D., Abteilungsleiter in einem Großunternehmen, erleidet 48-jährig einen Mediateilinfarkt links. Es resultiert eine mittelschwere Sprechapraxie ohne begleitende aphasische oder dysarthrische Symptomatik (reine Sprechapraxie). In der Spontansprache wie auch bei der Diagnostik mit strukturierten Wort- und Satzlisten fallen viele Lautbildungsfehler sowie eine ausgeprägte Verlangsamung und Monotonie (silbisches Sprechen) auf. Auf Lautfehler folgen oft mühsame und langwierige Korrekturversuche. Eine hohe Sprechanstrengung äußert sich in sichtbarer Anspannung im gesamten Gesichts- und Halsbereich, in übertrieben großen Artikulationsbewegungen sowie in Hochatmung und gepresster Stimmqualität. Die Verständlichkeit des Patienten ist dabei nahezu unbeeinträchtigt. Beruflich kann Herr D. ca. ein Jahr nach dem Ereignis wieder eine Teilzeittätigkeit in seinem früheren Unternehmen aufnehmen. Aufgrund der Sprechstörung ist es ihm jedoch nicht mehr möglich, in die Leitungsposition zurückzukehren, da diese unter anderem mit regelmäßigen Vortragstätigkeiten verbunden ist.

Die Funktionsstörungen haben typischerweise auch Auswirkungen auf die Prosodie.

\section{Störungen der Sprechatmung:}

- häufiges Zwischenatmen,

- hörbar angestrengte Einatmung,

- Sprechen auf Restluft,

- Ursachen:

- infolge von Schwäche, Rigidität oder Dyskoordination von abdominaler und thorakaler Atmungsmuskulatur oder

- infolge eines erhöhten Luftverlusts an der Glottis (Hypoadduktion), an der velopharyngealen Pforte (Gaumensegelinsuffizienz) oder bei der Artikulation (unvollständige Verschluss- oder Engebildung).

\section{Störungen der Sprechstimme:}

- zu leise/zu laute Stimme infolge zu geringen oder unkontrollierten Ausatmungsdrucks und/oder ineffizienter laryngealer Phonationsmechanismen,

- erniedrigte/erhöhte Stimmlage und raue/behauchte/gepresste Stimmqualität oder Aphonie infolge einer erhöhten, reduzierten oder unregelmäßigen Tonisierung oder einer Hyper-/Hypoadduktion der Stimmlippen,

- abrupte Änderungen von Tonhöhe/Lautstärke oder Stimmqualität infolge einer Dyskoordination von Adduktionsgrad und Spannung der Stimmlippen,

- Stimmtremor. 


\section{FALLBEISPIEL}

\section{Fall 3: Dysarthrie bei Zerebralparese}

Frau L., 21 Jahre, wurde in der 25. Schwangerschaftswoche geboren und erlitt postnatal eine periventrikuläre Hirnblutung. Im 1. Lebensjahr manifestierte sich eine Bewegungsstörung. Die Patientin zeigt eine rechtsbetonte spastische Tetraparese mit athetotischem Anteil. Zudem liegen eine kognitive Behinderung und eine Visusstörung vor. Die Patientin lebt in einem Wohnheim und arbeitet in einer Werkstatt für behinderte Menschen.

Im Vordergrund der Sprechstörung stehen ein erheblich reduziertes Sprechtempo (stark verlangsamte Artikulationsbewegungen und übermäßige Pausen) sowie Beeinträchtigungen der Stimmstabilität (v. a. Tonhöhen- und Lautstärkeschwankungen). Des Weiteren ist das Sprechen durch eine auffällige Stimmqualität, eine reduzierte Artikulationsschärfe sowie Monotonie gekennzeichnet. Das Bild entspricht dem Syndrom einer hyperkinetischen Dysarthrie.

Aufgrund der Dysarthrie ist die Verständlichkeit von Frau K. erheblich eingeschränkt. Das Sprechen der Patientin wird darüber hinaus als stark auffallend und unnatürlich beschrieben.

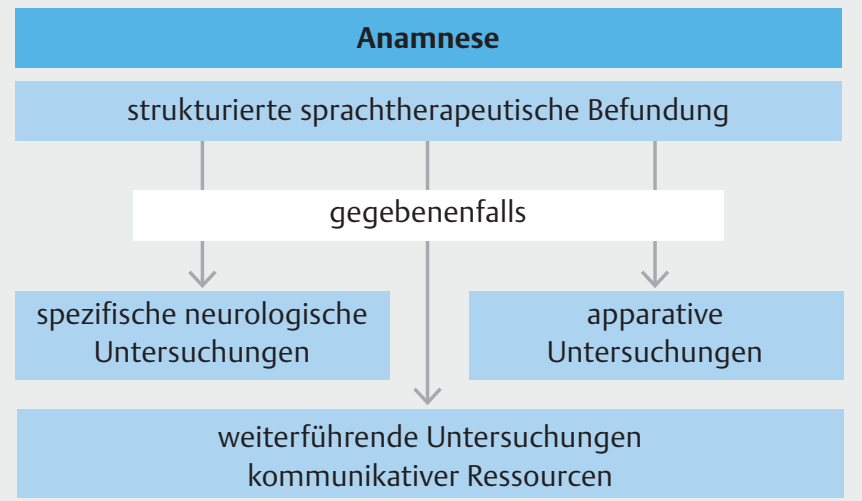

- Abb. 2 Diagnostisches Vorgehen bei neurogenen Sprechstörungen (Dysarthrie, Sprechapraxie) (Quelle: Ziegler W, Staiger A. Dysarthrie und Sprechapraxie. In: Diener H-C, Steinmetz H, Kastrup O, Hrsg. REFERENZ Neurologie. Stuttgart: Thieme; 2019: 112-119)

\section{Störungen der Artikulation und der nasalen Reso-} nanz:

- unpräzise Konsonanten-/Vokalartikulation infolge von Bewegungsstörungen von Unterkiefer, Zunge, Lippen und Gaumensegel bzw. mangelnder interartikulatorischer Koordination,

- Hypernasalität und nasaler Luftverlust infolge einer Gaumensegelparese.

\section{Störungen der Prosodie:}

- reduziertes Artikulationstempo infolge verlangsamter Artikulationsbewegungen,
- erhöht erscheinendes Artikulationstempo infolge hypokinetischer Artikulationsbewegungen (vor allem bei Morbus Parkinson),

- unflüssiges Sprechen infolge vermehrter Sprechpausen (z. B. durch häufiges Einatmen),

- Laut-/Silbenwiederholungen (z. B. stotterähnliche Symptome bei Morbus Parkinson),

- monotones Sprechen (z. B. infolge einer verringerten Fähigkeit zur Modulation der Stimmlippenspannung),

- silbisches („Silbe für Silbe“) Sprechen, z. B. als Kompensationsmechanismus bei ataktischer Dysarthrie.

\section{Merke}

Die Symptome der Atem-, Stimm- und Artikulationsfunktionen beeinflussen sich gegenseitig. Beispielsweise wird der nasale Luftverlust infolge einer Gaumensegelparese typischerweise eine Störung der Sprechatmung (häufige Zwischenatmungen) und der Artikulation (v. a. Laute, die kritisch von oralem Luftdruck abhängen) nach sich ziehen.

\section{Merke}

Die Symptome von Sprechapraxie und Dysarthrie können dazu führen, dass die Patienten nicht mehr ausreichend gut verstanden werden (reduzierte/aufgehobene Verständlichkeit) oder ihr Sprechen als auffällig und unnatürlich empfunden wird.

\section{Diagnostik}

\section{Diagnostisches Vorgehen}

Die spezifische Diagnostik der neurologischen Sprechstörungen zählt zum Aufgabengebiet der sprachtherapeutischen Berufsgruppen. Sie dient

- dem Nachweis einer sprechmotorischen Störung,

- der Feststellung des Störungstyps (Sprechapraxie, Dysarthriesyndrom),

- der Quantifizierung des Schweregrads,

- der Beschreibung des Störungsmusters (d. h. der relativen Anteile von Atmung, Phonation, Artikulation, Resonanz und Prosodie an der Sprechstörung),

- der Planung sprachtherapeutischer Versorgungsmaßnahmen,

- der Verlaufskontrolle sowie

- in einigen Fällen auch der neurologischen Diagnostik (z. B. Früherkennung einer beginnenden Parkinson-Erkrankung).

Das diagnostische Vorgehen baut auf vorliegenden neurologischen Befunden auf und mündet gegebenenfalls in Empfehlungen weiterführender neurologischer, phoniatrischer oder auch sprachtherapeutischer Untersuchungen. Das Kernstück der sprachtherapeutischen Diagnostik bildet eine eingehende systematische Un- 
tersuchung der Merkmale der Sprechstörung („sprachtherapeutische Untersuchung“; s. > Abb.2).

\section{Anamnese}

Das Vorgehen bei der Anamnese von Sprechapraxie und Dysarthrie entspricht überwiegend dem bei Aphasie (s.o.). Für sprechmotorische Störungen relevante Aspekte betreffen darüber hinaus:

- den Erkrankungsverlauf:

- bei Sprechapraxie: initialer Mutismus nach dem Schlaganfall? Dauer? (als prognostischer Faktor),

- zusätzliche Beeinträchtigungen der Sprache (Aphasie),

- nicht mit der neurologischen Erkrankung assoziierte Faktoren, die Einfluss auf das Sprechen haben,

- nicht-neurologische Erkrankungen der Atmungs-, Stimm- und Artikulationsorgane (z. B. chronisch obstruktive Lungenerkrankung, funktionelle Stimmstörung, Gewebeschädigungen von Artikulationsorganen nach Tumorresektion),

- Hörverlust (insbesondere bei beeinträchtigter Lautstärkeregulation),

- prämorbid bestehende Sprechstörungen (Artikulationsstörungen, Stottern, Poltern),

- Nikotinabusus („Raucherstimme“),

- idiosynkratrische Varianten (z. B. Dialekt).

\section{Strukturierte sprachtherapeutische Befundung}

Sprechapraxie: Die Symptomatik der Sprechapraxie (Störungsdimensionen Lautbildung, Prosodie und Sprechverhalten) kann durch auditive Beurteilung geeigneter Sprechproben bestimmt werden.

- Spontansprachproben (Interview) haben den Vorteil hoher Sensitivität und hoher ökologischer Validität. Sie haben den Nachteil, dass die sprechapraktische Symptomatik durch aphasische Symptome (z. B. Wortfindungsstörungen) maskiert sein kann. Der Schweregrad der Störung muss anhand auditiver Bewertungsskalen bestimmt werden. Etablierte reliable und valide Instrumente liegen dafür allerdings noch nicht vor.

- Nachsprechtests mit systematischen Wortlisten gestatten eine zuverlässigere Bestimmung sprechapraktischer Fehler, eine Quantifizierung des Störungsschweregrads und eine Erfassung der Faktoren, die die Symptomatik beeinflussen (z. B. Wortlänge, Silbenkomplexität, Wortfrequenz, Silbenfrequenz). Sie bilden das Ausmaß der sprechapraktischen Redeunflüssigkeit allerdings nur unvollständig ab. Die Bewertung erfolgt wortweise und liefert damit eine Quantifizierung des Störungsschweregrads [23].

Punktuell können auch akustische Sprachsignalanalyseverfahren eingesetzt werden, um einzelne Sprechparameter objektiv zu messen (s.u.).
Dysarthrie: Die Dysarthriediagnostik beinhaltet eine systematische Beurteilung der einzelnen Funktionskreise des Sprechens und ihres Zusammenwirkens:

- Sprechatmung:

- Einatmungshäufigkeit,

- Atmungsmuster,

- Phonation (Sprechstimme):

- Sprechlautstärke,

- Sprechtonhöhe,

- Stimmqualität,

- Stimmstabilität,

- Artikulation:

- Kieferöffnung beim Sprechen,

- Differenziertheit der Konsonanten- und Vokalbildung,

- Resonanz: nasaler Stimmklang und/oder nasaler Luftverlust bei der Artikulation,

- Prosodie:

- Artikulationstempo,

- Redeflüssigkeit,

- Rhythmus und

- Modulation.

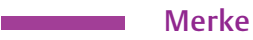

Goldstandard ist die auditive Diagnostik.

Sie erfordert Erfahrung in der Verwendung der auditiven Beurteilungskriterien und Expertenwissen über die Mechanismen der Sprechmotorik. Es existieren reliable und valide Untersuchungsinstrumente [24].

Die Beurteilung wird anhand von Sprechproben durchgeführt, die in einem Interview und durch lautes Lesen von Standardtexten, Nachsprechen von Sätzen oder kurze Erzählaufgaben erhoben werden können.

Zur Objektivierung der Befunde können unterstützend akustische Analyseverfahren eingesetzt werden (s.u.).

Zusätzliche Untersuchungen mit nicht-sprachlichen Aufgabenstellungen (z. B. Zunge herausstrecken, Wangen aufblasen etc.) können zur Prüfung der Hirnnervenfunktionen beitragen. Vokalhalteaufgaben („Sagen Sie möglichst lange „aaaaa...") können dazu dienen, das Vorliegen eines Stimmtremors zu prüfen und die Tremorfrequenz zu bestimmen. Solche Aufgaben geben allerdings keinen Aufschluss über die Art und den Schweregrad der Sprech- und Kommunikationsstörung.

Um die kommunikative Einschränkung zu beurteilen, kann u.a. eine Verständlichkeitsprüfung durchgeführt werden. Hierbei wird untersucht, wie gut ein Patient von anderen (Kommunikationspartnern) verstanden werden kann, beispielsweise mittels Schätzskalen („gar nicht verständlich“ bis „vollkommen verständlich“) oder über die Ermittlung des Anteils korrekt verstandener Silben einer Äußerung. 


\begin{abstract}
Merke
Sprechapraktische und dysarthrische Störungen haben in der Regel Auswirkungen auf kommunikative Aktivitäten und die soziale Teilhabe. Da die Messung an hohe methodische Anforderungen gebunden ist, sind geeignete Verfahren derzeit noch nicht in der klinischen Routinediagnostik verankert.
\end{abstract}

\section{Apparative Diagnostik}

Akustische Sprachsignalanalysen: Zur Objektivierung von Befunden können die Sprechproben sprechapraktischer und dysarthrischer Patienten mithilfe von Methoden der akustischen Sprachsignalverarbeitung analysiert werden:

- Stimmparameter (z. B. Sprechstimmlage, Stimmqualität),

- Artikulationsparameter (z. B. Vokalartikulation wie Vokalformanten) sowie

- Prosodieparameter (z. B. Artikulationstempo, Redefluss).

Akustische Analyseparameter haben eine höhere Sensitivität und eine höhere Reliabilität als auditive Urteile. Allerdings sind viele Parameter artefaktbehaftet und nur eingeschränkt valide. Für die zuverlässige Anwendung ist eine hohe phonetische Expertise erforderlich.

Bewegungsanalysen: Mittels verschiedener apparativer Methoden können Sprechbewegungen direkt oder indirekt registriert und analysiert werden, beispielsweise im Hinblick auf

- die Sprechatmung (z. B. induktive Plethysmografie oder Magnetometrie zur Messung der thorakalen und abdominalen Volumenänderungen),

- die Artikulation (Elektropalatografie [EPG] zur Erfassung der lingualen Kontaktmuster am harten Gaumen während der Konsonantenartikulation).

\section{HINTERGRUNDINFORMATION}

\section{Digitale Lösungen in der Dysarthriediagnostik}

Derzeit werden in zunehmendem Maße Studien publiziert, in denen mithilfe maschineller Lernalgorithmen versucht wird, auf der Grundlage kurzer digitaler Sprachaufnahmen diagnostische Entscheidungen zu treffen (z. B. Früherkennung degenerativer Erkrankungen). Bislang beruhen diese Studien ausnahmslos auf geringen Fallzahlen und beinhalten keine ausreichende Out-of-Sample Validierung, sodass zuverlässige klinische Anwendungen dieser Methoden noch nicht absehbar sind. Andere Entwicklungen zielen darauf ab, in Ergänzung zur klinischen Standarddiagnostik akustische und auditive Analyseverfahren als telediagnostische Web-Anwendungen anzubieten. Mit solchen Angeboten können technisch und infrastrukturell anspruchsvolle Methoden für einen großen Kreis von klinischen Anwendern verfügbar gemacht werden, z. B. valide Methoden für die Verständlichkeitsmessung [26].
Die Bedeutung dieser Verfahren für die klinische Diagnostik ist wegen ihres hohen technischen Aufwandes, der damit verbundenen Belastung für die Patienten und der teilweise eingeschränkten Aussagekraft der Messparameter nur gering.

\section{Elektromyografie:}

- Zungen-EMG: Zum Nachweis einer Denervierung oder von Fibrillationen bei Verdacht auf das Vorliegen einer Motoneuronerkrankung.

- Larynx-EMG (Mm. thyroarytaenoideus, cricothyroideus): in der Diagnostik von Stimmlippenparesen und in der EMG-geleiteten Botulinumtoxinbehandlung der spasmodischen Dysphonie [25].

Laryngoskopie: Bei auffälligen Stimmbefunden kann eine phoniatrische Kehlkopfuntersuchung angezeigt sein.

- Transorale Lupenlaryngoskopie: Inspektion von Hypopharynx und Larynx in Ruhe und bei der isolierten Phonation; vor allem zur Erkennung von Gewebeschädigungen und von peripheren Stimmlippenparesen, Hyperkinesen, Tremor und Myoklonien.

- Laryngostroboskopie: Zur Feinanalyse des Ablaufs der Stimmlippenschwingungen bei isolierter Phonation.

- Transnasale Videoendoskopie: Beobachtung der Kehlkopffunktion auch während des Sprechens.

\section{Merke}

Die auditive sprachtherapeutische Befundung stellt den derzeitigen Goldstandard in der Diagnostik von Dysarthrie und Sprechapraxie dar. Apparative Verfahren können die auditive Diagnostik im Hinblick auf spezifische Fragestellungen unter Umständen sinnvoll ergänzen.

\section{Therapie}

Sprachtherapeutische Maßnahmen

Die Auswahl geeigneter therapeutischer Vorgehensweisen bei neurogenen Sprechstörungen ist von individuellen Faktoren bestimmt, u.a.

- Grunderkrankung,

- Art der Sprechstörung (Sprechapraxie, Dysarthrie),

- Störungsmuster (Ausprägung und Schweregrad von Störungen der Artikulation, Resonanz, Phonation, Sprechatmung, Prosodie),

- neuropsychologische Begleitstörungen (z. B. Aphasie, bukkofaziale Apraxie),

- psychosoziale Faktoren.

Behandlungsziele lassen sich auf verschiedenen Ebenen definieren [13]:

- Funktion: Verbesserung sprechmotorischer Funktionen (z. B. Verbesserung von Artikulation und Phonation), 
- Aktivität und Teilhabe: Verbesserung kommunikativer Fähigkeiten (z. B. Erhöhung von Verständlichkeit und Sprechnatürlichkeit) und damit Verbesserung von Teilhabemöglichkeiten (z. B. aktivere Teilnahme an Gesprächen),

- Umweltfaktoren: Substitution und Umweltadaptation (z. B. Verbesserung der Kommunikation durch technische Kommunikationshilfen, Anpassung des sozialen/beruflichen Umfelds an veränderte Sprechbedingungen).

\section{Merke}

Aufgrund der großen Heterogenität der pathophysiologischen Bedingungen und der individuellen Störungsmuster sind einem standardisierten Verfahren enge Grenzen gesetzt.

Im Zentrum der sprachtherapeutischen Übungsbehandlung steht das intensive motorische Üben. Es stützt sich im Wesentlichen auf drei Säulen:

- Restitution beeinträchtigter sprechmotorischer Funktionen,

- Vermittlung von Kompensationsstrategien,

- Abbau/Vorbeugung von Fehlanpassungen.

Die Verbesserung nichtsprachlicher Bewegungsfunktionen der am Sprechen beteiligten Muskulatur stellt dabei kein primäres Behandlungsziel dar.

Sprechapraxie: Die Therapie der Sprechapraxie erfolgt üblicherweise im sprachtherapeutischen Übungssetting. Durch motorisches Üben wird eine Reorganisation sprechmotorischer Planungsprozesse angestrebt. Funktionsziele beinhalten die Verbesserung artikulatorischer und prosodischer Leistungen (z. B. Erarbeiten artikulatorischer Zielkonfigurationen, Verbesserungen des Redeflusses) sowie die Modifikation des Sprechverhaltens (z.B. Abbau von Sprechanstrengung). Die Übungsbehandlungen können durch Hilfsmittel und Biofeedback-Techniken ergänzt werden.

Etablierte Methoden der spezifischen Sprechapraxietherapie lassen sich in die folgenden Behandlungskategorien einteilen [27]:

- artikulatorisch-kinematische Verfahren: z. B. Minimalpaar-Training, PROMPT,

- auf Rhythmus- und Tempokontrolle beruhende Verfahren: z. B. Taktgeber/Tastbrett,

- Methoden der intersystemischen Reorganisation/ Fazilitierung: z. B. Unterstützung von Sprechbewegungen durch lautspezifische Handgesten,

- unterstützte und alternative Kommunikation (AAC) bei Patienten mit stark eingeschränkter mündlicher Kommunikationsfähigkeit/Mutismus,

- Beratung und Angehörigenarbeit.

\section{Merke}

Für die Klassen der artikulatorisch-kinematischen Verfahren und auf Rhythmus- und Tempokontrolle beruhenden Verfahren ist die Wirksamkeit durch mehrere Studien belegt, wenngleich Studien mit höherem Evidenzgrad noch ausstehen [27].

Dysarthrie: Im Zentrum der Maßnahmen steht die sprachtherapeutische Übungsbehandlung (inklusive feedbackgestützte Trainingsmethoden):

- durch intensives motorisches Üben:

- Restitution beeinträchtigter sprechmotorischer Funktionen: z. B. Maßnahmen zur willentlichen Modifikation der Atemphasen, zur Verbesserung des Glottisschlusses oder zur Verbesserung der lingualen Engebildung,

- Vermittlung von Kompensationsstrategien: z. B. Reduktion des Sprechtempos zur Erhöhung der Sprechverständlichkeit,

- Abbau/Vorbeugung von Fehlanpassungen: z. B. Modifikation von übermäßiger Kieferöffnungsweite;

- Hilfsmittel und prothetische Hilfen:

- zur Verlangsamung des Sprechtempos: z. B. Taktgeber/Tastbrett, elektronische Sprachverzögerer,

- zur Steigerung der Sprechlautstärke: z. B. tragbare Stimmverstärker, Maskierungsgeräte,

- zur Verbesserung artikulatorischer Funktionen bei Störungen der Unterkieferkontrolle: Beißblock,

- zur Verhinderung des nasalen Luftverlusts bei Gaumensegelparese: Gaumensegelprothese, Nasalanzventil;

- unterstützte und alternative Kommunikation (AAC) bei Patienten mit schwerer Dysarthrie, z. B. bei Zerebralparese, amyotropher Lateralsklerose, schwerem Schädel-Hirn-Trauma;

- Beratung und Angehörigenarbeit.

\section{Merke}

Therapeutische Übungen sollten sich möglichst an natürlichen sprechmotorischen Abläufen und an Prinzipien motorischen Lernens orientieren. Es stehen zahlreiche therapeutische Verfahren zur Verfügung; Beispiel: Lee-Silverman-Voice-Treatment (LSVT ${ }^{\circledR}$ ) [28].

Nach einer von der Deutschen Gesellschaft für Neurologie (DGN) entwickelten Leitlinie [29] liegen im Bereich der sprachtherapeutischen Übungsbehandlungen Wirksamkeitsnachweise ausschließlich für das Lee-Silverman Voice Treatment (LSVT) bei Patienten mit Morbus Parkinson vor. 


\section{THERAPIE}

Verordnung von Sprachtherapie (Heilmittelkatalog)

Nach Heilmittelkatalog: Ziffer 2.5 Störungen der Sprechmotorik, Diagnosegruppe SP6 Dysarthrie/Sprechapraxie.

- Heilmittel: Stimm-, Sprech- und Sprachtherapie 30/45 oder 60 Minuten mit dem Patienten, je nach konkretem Störungsbild und Belastbarkeit.

- Verordnungsmenge: Erst-VO: bis zu 10×/VO. Folge-VO: bis zu $20 \times / V O$. Gesamtverordnungsmenge des Regelfalls: bis zu 60 Einheiten.

- Frequenzempfehlung: mindestens $1 \times$ wöchentlich.

Es gibt zahlreiche Hinweise dafür, dass sich eine hohe Übungsfrequenz günstig auf motorisches Lernen auswirkt. Ungeklärt ist bislang, wie hoch die Behandlungsfrequenz und -dauer für die beste Wirksamkeit der Therapie von Sprechapraxie und Dysarthrie genau sein sollte.

Evidenzbasierte Therapieempfehlungen existieren auBerdem für die Behandlung von Resonanzstörungen aufgrund einer Gaumensegelparese mittels einer Gaumensegelprothese.

\section{Pharmakotherapie}

Bislang steht keine pharmakologische Therapie zur spezifischen Beeinflussung der Dysarthrie oder Sprechapraxie zur Verfügung.
Pharmakologische Therapien zur Behandlung der Grunderkrankung gehen nicht unbedingt mit Verbesserungen sprechmotorischer Leistungen einher. Studienergebnisse zu Auswirkungen einer L-Dopa-Substitutionstherapie auf die Dysarthrie bei Morbus Parkinson sind heterogen [26].

Als etabliertes Verfahren gilt die Injektion von Botulinumtoxin in den M. thyroarytaenoideus bei spasmodischer Dysphonie. Botulinumtoxin findet auch bei der Behandlung von oromandibulären Dystonien sowie bei mandibulärem, velopharyngealem und laryngealem Tremor Anwendung.

\section{Operative Therapie}

Tiefe Hirnstimulation (THS) zur Reduktion der motorischen Leitsymptome bei Morbus Parkinson: Trotz stimulationsbedingter Verbesserungen der Extremitätenmotorik lassen sich keine vergleichbar positiven Effekte auf das Sprechen erwarten [26]. Einige Faktoren stehen sogar in Zusammenhang mit Verschlechterungen des Sprechens (z.B. operationsbedingte Mikroläsionen, Platzierung der Elektroden, ungünstige Stimulationsparameter).

- Tab.2 Differenzialdiagnosen der Aphasie.

\begin{tabular}{|c|c|}
\hline Differenzialdiagnose & Bemerkung \\
\hline $\begin{array}{l}\text { Sprachstörung nach rechtshemisphärischer } \\
\text { Läsion }\end{array}$ & $\begin{array}{l}\text { Störung betrifft eher den Gebrauch der Sprache im kommunikativen Kontext („Pragmatik“) } \\
\text { und Textebene } \\
\text { Betroffen sind z. B. Verarbeitung von Ironie, Mehrdeutigkeit und indirekten Aussagen } \\
\text { Linguistisches Wissen meist nicht gestört }\end{array}$ \\
\hline psychiatrische Erkrankung & Denkstörung bei Psychose \\
\hline Alzheimer-Demenz & initial keine Sprachstörung, sondern vor allem Einschränkung des episodischen Gedächtnisses \\
\hline Sprechapraxie & $\begin{array}{l}\text { s. Tab. 3, bei isoliertem Auftreten keine Einschränkung des Sprachverständnisses, keine } \\
\text { Störungen des Schreibens oder Lesens }\end{array}$ \\
\hline
\end{tabular}

- Tab.3 Differenzialdiagnosen der Sprechapraxie.

\begin{tabular}{|c|c|}
\hline Differenzialdiagnose & Bemerkung \\
\hline $\begin{array}{l}\text { Phonologische Störung } \\
\text { aphasisch bedingte Störungen der Lautbildung } \\
\text { („phonematische Paraphasien“) }\end{array}$ & $\begin{array}{l}\text { für die Diagnose aphasisch-phonologische Störung und gegen die Diagnose Sprechapraxie } \\
\text { spricht } \\
\text { " relativ flüssiges Artikulieren } \\
\text { " das Fehlen erkennbar motorischer Anzeichen fehlerhafter Lautbildung und gestörter Laut- } \\
\text { übergänge (Lautfehler sind „wohlartikuliert“) }\end{array}$ \\
\hline Dysarthrie & s. $>$ Tab. 4 \\
\hline bukkofaziale Apraxie & $\begin{array}{l}\text { der Begriff „bukkofaziale Apraxie“ bezeichnet keine Sprechstörung, sondern die fehlerhafte } \\
\text { Ausführung nonverbaler motorischer Aktionen, imitatorisch oder auf Anweisung (z. B. „Mund } \\
\text { öffnen“, „Zunge herausstrecken“, „, Summen“ etc.) } \\
\text { die Störung wird meist erst bei klinischer Prüfung bemerkt } \\
\text { Sprechapraxie und bukkofaziale Apraxie können dissoziieren }\end{array}$ \\
\hline
\end{tabular}


$\checkmark$ Tab. 4 Differenzialdiagnosen der Dysarthrie.

\begin{tabular}{|l|l|}
\hline Differenzialdiagnose & Bemerkung \\
\hline Sprechapraxie & $\begin{array}{l}\text { für die Diagnose Sprechapraxie und gegen die Diagnose Dysarthrie spricht: } \\
\text { - das Vorliegen einer fokalen linkshemisphärischen kortikalen Schädigung }\end{array}$ \\
\hline $\begin{array}{l}\text { - inkonstantes, inkonsistentes Fehlermuster und das Auftreten störungsfreier } \\
\text { Passagen } \\
\text { das Auftreten von phonematischen Fehlern (z. B. Lautersetzungen) }\end{array}$ \\
\hline $\begin{array}{l}\text { Dysglossie } \\
\text { Artikulationsstörung infolge von angeborenen } \\
\begin{array}{l}\text { Fehlbildungen, Verletzungen oder Tumoren } \\
\text { der Artikulationsorgane; analog: Dysphonie } \\
\text { infolge laryngealer Gewebeschädigungen }\end{array}\end{array}$ & $\begin{array}{l}\text { Klärung durch Ausschluss einer neurologischen Erkrankung und Nachweis einer } \\
\text { strukturellen Schädigung der Sprechorgane }\end{array}$ \\
\hline
\end{tabular}

\section{Spezifisch sprechunterstützende Operationsverfah-} ren:

- Fazialisnervenplastik bei peripherer Fazialisparese,

- operative Verfahren bei velopharyngealer Insuffizienz (Velopharyngoplastik, Levatorplastik, Pharyngoplastik),

- Laryngoplastik bei peripheren Stimmlippenparesen.

\section{Differenzialdiagnosen}

Die Differenzialdiagnosen der Aphasie, der Sprechapraxie und der Dysarthrie fassen > Tab.2, Tab.3 u. - Tab. 4 zusammen.

\section{KERNAUSSAGEN}

- Aphasie, Sprechapraxie und Dysarthrie stellen eigenständige klinische Entitäten dar. Ihre Unterscheidung ist zentral für die Wahl des Behandlungsansatzes.

- Aphasien sind nicht Denk-, sondern Sprachstörungen. Sie können sowohl die mündliche als auch die schriftliche Kommunikationsfähigkeit beeinträchtigen und zu weitreichenden Einschränkungen in Selbständigkeit und Teilhabe führen.

- In der Testung auf Aphasie sollten immer auch rezeptive sprachliche Leistungen überprüft werden.

- Sprachtherapie bei Aphasie ist auch jenseits von 1 Jahr Krankheitsdauer nachweislich wirksam.

- Weder Alter noch Krankheitsdauer haben einen Einfluss auf das Verbesserungspotenzial bei vaskulär bedingter chronischer Aphasie.

- Wirksamkeitsnachweise liegen insbesondere für intensive Therapieansätze bei Aphasie vor.
- Dysarthrien stellen die häufigste Form neurogener Kommunikationsstörungen dar.

- Indem sie die Verständlichkeit, die Natürlichkeit und die kommunikative Effizienz beim Sprechen reduzieren, können Sprechapraxie und Dysarthrie zu Beeinträchtigungen der Kommunikationsfähigkeit und zu Einschränkungen der sozialen Teilhabe führen.

- Auditive, von Sprachtherapeuten durchgeführte Analyseverfahren bilden den Goldstandard in der Diagnostik neurogener Sprechstörungen. Eine Ergänzung durch kommunikationsorientierte Messverfahren sowie durch objektive, insbesondere akustische Analysen kann sinnvoll sein.

- Es existieren zahlreiche etablierte Therapieansätze für Dysarthrie und Sprechapraxie. Für die meisten Verfahren stehen Wirksamkeitsbelege durch randomisierte kontrollierte Studien mit großen Fallzahlen jedoch noch aus. 


\section{Danksagung}

Teile dieses Artikels bauen auf dem Beitrag von Ziegler, W. \& Staiger, A. (2019) „Dysarthrie und Sprechapraxie“ In: Diener, H.-C., Steinmetz, H., Kastrup, O. (eds.), REFERENZ Neurologie (pp. 112-119). Thieme auf. Wir bedanken uns bei Theresa Schölderle und Elisabet Haas für ihren Beitrag zu dem Fallbeispiel für Dysarthrie. Vielen Dank an Wolfram Ziegler und Katharina Lehner für die kritische Durchsicht des Manuskripts und die wertvollen Verbesserungsvorschläge.

Interessenkonflikt

Erklärung zu finanziellen Interessen

Forschungsförderung erhalten: ja, von einer anderen Institution; Honorar/geldwerten Vorteil für Referententätigkeit erhalten: nein; Bezahlter Berater/interner Schulungsreferent/Gehaltsempfänger: nein; Patent/Geschäftsanteile/ Aktien (Autor/Partner, Ehepartner, Kinder) an Firma (NichtSponsor der Veranstaltung): nein; Patent/Geschäftsanteile/ Aktien (Autor/Partner, Ehepartner, Kinder) an Firma (Sponsor der Veranstaltung): nein.

\section{Erklärung zu nichtfinanziellen Interessen}

Die Autorinnen/Autoren geben an, dass kein Interessenkonflikt besteht.

\section{Autorinnen/Autoren}

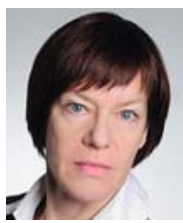

\section{Annette Baumgärtner}

Prof. Annette Baumgärtner PhD ist promovierte Logopädin und leitet seit $2017 \mathrm{ge}$ meinsam mit Prof. Katharina Röse den berufsbegleitenden Studiengang Ergotherapie/ Logopädie an der Sektion Medizin der Universität zu Lübeck. Ihr wissenschaftlicher Schwerpunkt liegt auf Diagnostik und Therapie der Aphasie.

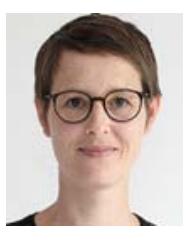

\section{Anja Staiger}

Dr. Anja Staiger ist Logopädin und Klinische Linguistin. Sie ist als wissenschaftliche Mitarbeiterin in der Entwicklungsgruppe Klinische Neuropsychologie (EKN), Institut für Phonetik und Sprachverarbeitung der LMU München, tätig. Ihre Forschungsschwerpunkte sind neurogene Sprechstörungen (Sprechapraxie, Dysarthrie).

\section{Korrespondenzadresse}

\section{Prof. Annette Baumgärtner}

Sektion Medizin

Institut für Gesundheitswissenschaften

Universität zu Lübeck

Ratzeburger Allee 160

23562 Lübeck

annette.baumgaertner@uni-luebeck.de
Wissenschaftlich verantwortlich gemäß Zertifizierungsbestimmungen

Wissenschaftlich verantwortlich gemäß Zertifizierungsbestimmungen für diesen Beitrag ist Prof. med. Hans-Christoph Diener, Essen.

Literatur

[1] Worrall L, Sherratt S, Rogers P et al. What people with aphasia want: Their goals according to the ICF. Aphasiology 2011; 25: 309-322

[2] Huber W, Poeck K, Springer L. Klinik und Rehabilitation der Aphasie. Stuttgart: Thieme; 2013

[3] National Institute on Deafness and Other Communication Disorders. NIDCD fact sheet: Aphasia [PDF] [NIH Pub. No. 97-4257]. 2015: Abgerufen am 20.3.2020 auf https:// www.nidcd.nih.gov/health/aphasia

[4] Knecht S, Hesse S, Oster P. Aphasia in acute stroke: incidence, determinants, and recovery. Ann Neurol 1995; 38: 659-666

[5] Pedersen PM, Jorgensen HS, Nakayama $\mathrm{H}$ et al. Aphasia in acute stroke: incidence, determinants, and recovery. Ann Neurol 1995; 38: 659-666

[6] Lazar RM, Minzer B, Antoniello D et al. Improvement in aphasia scores after stroke is well predicted by initial severity. Stroke 2010; 41: 1485-1488

[7] Breitenstein C, Grewe T, Flöel A et al. Intensive speech and language therapy in patients with chronic aphasia after stroke: a randomised, open-label, blinded-endpoint, controlled trial in a health-care setting. Lancet 2017; 389: $1528-1538$

[8] Meinzer M, Mohammadi S, Kugel H et al. Integrity of the hippocampus and surrounding white matter is correlated with language training success in aphasia. Neuroimage 2010; 53: 283-290

[9] Huber W, Poeck K, Weniger D et al. Aachener Apasie Test (AAT). Göttingen: Hogrefe; 1983

[10] Nobis-Bosch R, Rubi-Fessen I, Biniek R et al. Diagnostik und Therapie der akuten Aphasie. Stuttgart: Georg Thieme Verlag; 2012

[11] Richter K, Wittler M, Hielscher-Fastabend M. Bielefelder Aphasie Screening (BiAS). Zur Diagnostik akuter Aphasien. Hofheim: NAT-Verlag; 2006

[12] Kalbe E, Reinhold N, Ender U et al. Aphasie Check Liste. Muttenz: ProLog; 2010

[13] DIMDI. Internationale Klassifikation der Funktionsfähigkeit, Behinderung und Gesundheit. Genf: WHO; 2005

[14] Baumgärtner A, Gröne B, Maser I. Intensive Aphasietherapie: Evidenz und Umsetzung im ambulanten und (teil-)stationären Setting. Neurol Rehabil 2018; 24: 99-109

[15] Volkmer A, Rogalski E, Henry M et al. Speech and language therapy approaches to managing primary progressive aphasia. Pract Neurol 2019: doi:10.1136/practneurol2018-001921

[16] Baumgärtner A. Intensität in der Aphasietherapie. In: Grötzbach $\mathrm{H}$. Intensität in der Sprachtherapie. Idstein: Schulz-Kirchner; 2017: 41-68

[17] Zhang X, Shu B, Zhang D et al. The efficacy and safety of pharmacological treatments for post-stroke aphasia. CNS Neurol Disorders Drug Targets 2018; 17: 509-521 
[18] Norise C, Hamilton RH. Non-invasive brain stimulation in the treatment of post-stroke and neurodegenerative aphasia: parallels, differences, and lessons learned. Frontiers Hum Neurosci 2017; 10: 675

[19] Elsner B, Kugler J, Pohl M et al. Transcranial direct current stimulation (tDCS) for improving aphasia in adults with aphasia after stroke. Im Internet (Stand 28.03.2020): Cochrane Database Syst Rev 2019: https://www.cochranelibrary. com/cdsr/doi/10.1002/14651858.CD009760.pub4/full

[20] Heikkinen PH, Pulvermüller F, Mäkelä JP et al. Combining rTMS With Intensive Language-Action Therapy in Chronic Aphasia: a randomized controlled trial. Front Neurosci 2019; 12: 1036. doi:10.3389/fnins.2018.01036

[21] Brady MC, Kelly H, Godwin J et al. Speech and language therapy for aphasia following stroke. Cochrane database of systematic reviews 2016: doi:10.1002/14651858. CD000425.pub4

[22] Ziegler W, Aichert I, Staiger A. Sprechapraxie: Grundlagen, Diagnostik, Therapie. Heidelberg: Springer; 2020

[23] Liepold M, Ziegler W, Brendel B. Hierarchische Wortlisten. Ein Nachsprechtest für die Sprechapraxiediagnostik. Dortmund: Borgmann; 2003

[24] Ziegler W, Schölderle T, Staiger A et al. BoDys: Bogenhausener Dysarthrieskalen. Göttingen: Hogrefe; 2018

[25] Blitzer A, Crumley RL, Dailey SH et al. Recommendations of the Neurolaryngology Study Group on laryngeal electromyography. Otolaryngol Head Neck Surg 2009; 140: $782-$ 793. e786
[26] Lehner K, Ziegler W. Crowdbasierte Methoden in der Diagnostik neurologischer Sprechstörungen. Aphasie Verwandte Gebiete 2019; 2: 28-33

[27] Ballard KJ, Wambaugh JL, Duffy JR et al. Treatment for acquired apraxia of speech: a systematic review of intervention research between 2004 and 2012. Am J Speech Language Pathol 2015; 24: 316-337

[28] Ramig L, Halpern A, Spielman J et al. Speech treatment in Parkinson"s disease: Randomized controlled trial (RCT). Move Disord 2018; 33: 1777-1791

[29] Ackermann $\mathrm{H}$ et al. Neurogene Sprechstörungen (Dysarthrien), S1-Leitlinie. In: Leitlinien für Diagnostik und Therapie in der Neurologie.Deutsche Gesellschaft für Neurologie; 2018: Online (Stand 28.03.2020): www.dgn.org/leitlinien

[30] Nebel A, Deuschl G. Dysarthrie und Dysphagie bei Morbus Parkinson. Stuttgart: Georg Thieme Verlag; 2017

Bibliografie

DOI https://doi.org/10.1055/a-0966-0974 Neurologie up2date 2020; 03: 155-173 (c) Georg Thieme Verlag KG Stuttgart · New York ISSN 2511-3453 


\section{Punkte sammeln auf CME.thieme.de}

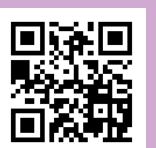

Diese Fortbildungseinheit ist in der Regel 12 Monate online für die Teilnahme verfügbar.

Den genauen Einsendeschluss finden Sie unter https://cme.thieme.de.

Sollten Sie Fragen zur Online-Teilnahme haben, finden Sie unter https://cme.thieme.de/hilfe

eine ausführliche Anleitung. Wir wünschen viel Erfolg beim Beantworten

der Fragen!

Unter https://eref.thieme.de/CXDHUAE oder über den QR-Code kommen Sie direkt zur Startseite des Wissenstests.

VNR 2760512020158724605

\section{Frage 1}

Welche Aussage zu Aphasien ist richtig?

A Aphasien sind vorrangig durch Störungen der Sprachproduktion gekennzeichnet.

B Aphasien sind vor allem Ausdruck einer Denkstörung.

C Aphasien können alle rezeptiven und expressiven sprachlichen Fähigkeiten betreffen.

D Aphasien gehen in der Regel mit Denkstörungen einher.

E Aphasien können nur die schriftsprachlichen Fähigkeiten betreffen.

\section{Frage 2}

Welche Aussage zur Ätiologie bei Aphasie ist richtig?

A Aphasie lässt sich eindeutig den non-demenziellen Erkrankungen zuordnen.

B Aphasie kann auch bei demenziellen Erkrankungen auftreten.

C Aphasie tritt zu gleichen Anteilen bei demenziellen und non-demenziellen Ätiologien auf.

D Schädel-Hirn-Trauma ist die häufigste Ätiologie bei Aphasie.

E Der Krankheitsverlauf lässt keine Rückschlüsse auf die Ätiologie bei Aphasie zu.

\section{Frage 3}

Wodurch ist die Prognose bei Aphasie nach Schlaganfall gekennzeichnet?

A Die Krankheitsdauer ist ein entscheidender Faktor für das Verbesserungspotenzial bei Aphasie.

B Initial schwergradige Aphasien haben grundsätzlich eine ungünstige Prognose.

C Eine vollständige Rückbildung der Symptomatik ist vor allem für initial leichtgradige Aphasien zu erwarten.

D Für Aphasiepatienten mit Re-Insult ist die Prognose ungünstig.
E Ein höheres Bildungsniveau wirkt sich positiv auf das Verbesserungspotenzial aus.

\section{Frage 4}

Therapieprinzipien bei Aphasie: Welche Aussage ist richtig?

A Überschießende Sprachproduktion sollte gefördert werden.

B Die Therapie fokussiert vor allem auf gestörte sprachliche Mechanismen.

C Die Therapie fokussiert vor allem auf Umweg- und Kompensationsstrategien.

D Sprachliche Übungen werden in immer gleicher Abfolge durchgeführt.

E Übungen zum Sprachverständnis sollten vor sprachlichproduktiven Übungen erfolgen.

\section{Frage 5}

Nur eine der Aussagen zur Evidenzlage in der Aphasietherapie ist richtig. Welche?

A Es liegen Wirksamkeitsnachweise für niederfrequente Therapieansätze vor.

B Eine Therapie bei chronischer Aphasie ist nicht evidenzbasiert.

C Die Therapie in der frühen Phase nach Aphasie ist evidenzbasiert.

D Es existieren noch keine Wirksamkeitsnachweise für Sprachtherapie bei Aphasie.

E Intensivtherapie bei chronischer Aphasie ist nachweislich wirksam. 


\section{Punkte sammeln auf CME.thieme.de}

Fortsetzung ...

\section{Frage 6}

Welche Aussage zur Sprechapraxie ist richtig?

A Eine schwere und persistierende Sprechapraxie resultiert in der Regel aus bilateralen Hirnläsionen.

B Der Sprechapraxie liegen beeinträchtigte sensorische Funktionen zugrunde.

C Typischerweise sind bei Sprechapraxie die Sprechstimme und Sprechatmung mit betroffen.

D Die Sprechapraxie ist meistens mit aphasischen Sprachstörungen assoziiert.

E Das Fehlermuster bei Sprechapraxie ist durch eine geringe Variabilität gekennzeichnet.

\section{Frage 7}

Welche Aussage zur Dysarthrie ist richtig?

A Dysarthrien liegen Störungen der Planung von Sprechbewegungen zugrunde.

B Dysarthrien liegen Störungen der Ausführung von Sprechbewegungen zugrunde.

C Dysarthrien sind durch Beeinträchtigungen sprachlicher und sprechmotorischer Funktionen gekennzeichnet.

D Liegt eine Dysarthrie vor, ist die Sprechatmung nicht betroffen.

E Dysarthrien resultieren aus Schädigungen der sprachdominanten Hemisphäre.

\section{Frage 8}

Dysarthriediagnostik: Welche Aussage ist zutreffend?

A Die Diagnostik erfordert die systematische Beurteilung der Funktionskreise Sprechatmung, Sprechstimme und Artikulation.

B Objektive Methoden (v. a. Methoden der akustischen Signalanalyse) sind auditiven Untersuchungsverfahren grundsätzlich vorzuziehen.

C Beobachtungen nicht-sprachlicher Bewegungsfunktionen der am Sprechen beteiligten Muskulatur liefern wichtige Informationen zu Art und Schweregrad der Sprechstörung.

D Zur Beurteilung der Kommunikationsfähigkeit sollte das Sprachverständnis geprüft werden.

E Für die Diagnostik der Dysarthrie stehen keine spezifischen Untersuchungsmethoden zur Verfügung.

\section{Frage 9}

Sprechapraxietherapie: Welche Aussage ist zutreffend?

A Derzeit gibt es noch keine Behandlungsverfahren für Sprechapraxie, deren Wirksamkeit durch Studien belegt wäre.

B Bei gleichzeitigem Bestehen einer Aphasie stellt die Verbesserung der sprechapraktischen Symptomatik kein eigenständiges Behandlungsziel dar.

C Durch sprechmotorisches Üben wird eine Reorganisation sprechmotorischer Planungsprozesse angestrebt.

D Die Therapie bei Sprechapraxie fokussiert sprachlich rezeptive Leistungen.

E Der Einsatz einer Gaumensegelprothese wirkt sich positiv auf die Artikulationsfähigkeit bei Sprechapraxie aus.

\section{Frage 10}

Ist ein Patient mit Dysarthrie oder Sprechapraxie in seiner Verständlichkeit beeinträchtigt, so kann er ...

A gesprochene Sprache (z. B. Äußerungen von Gesprächspartnern) nicht mehr ausreichend gut verstehen.

B keinen klaren Gedanken mehr fassen.

C seine Mitteilungsabsicht nicht mehr in schriftlicher Weise übermitteln.

D von Kommunikationspartnern aufgrund seiner Sprechstörung nicht mehr ausreichend gut verstanden werden.

E kulturspezifische Kommunikationsregeln nicht mehr einhalten. 